\title{
Recent Advancements in Laboratory X-ray Microscopes Enabling 3D and 4D Science
}

\author{
Arno Merkle and Jeff Gelb
}

Xradia, Inc., Pleasanton, CA, USA

In recent years, the microscopy community has seen a rapid increase in the availability of hard X-ray imaging instrumentation within central laboratories. The non-destructive nature of X-rays has made the technique widely appealing, with the potential for characterizing sample changes in "4D," delivering 3D microstructural information on physically the same sample over time, as a function of sequential processing conditions or experimental treatments. This has led to a new regime of functional studies, with applications spanning the life sciences, physical sciences, geosciences, and electronics industry, and is in a state of rapid expansion. [1-2]

This technique of X-ray microscopy (XRM) is currently evolving into standard practice for many disciplines and the technique is fueled by the historical developments that have taken place in the synchrotron facilities worldwide. Using reflective capillary condenser lenses coupled with diffractive Fresnel imaging objectives, beamlines in some facilities are now pushing the $10 \mathrm{~nm}$ resolution levels. [3] Laboratory instrumentation has grown out of these developments, and laboratory X-ray microscopes are now able to achieve resolutions on the order of $50 \mathrm{~nm}$, using analogous optical architectures to those in the synchrotrons (with obvious source differences). Commercially available nano-XRMs, such as the Xradia UltraXRM-L series, are transitioning this technology into the central laboratories (Figure 1), enabling unprecedented high-resolution studies of soft and hard materials alike without the need for synchrotron radiation. [4] The primary driver for this need is to focus on correlative and time-dependent (4D) experiments.

Micron-scale x-ray tomography is also commonplace in synchrotrons, but traditional laboratory microand nano-CT instrumentation has been lagging behind the synchrotron developments. This is due largely to the system geometry most often used by laboratory micro- and nano-CT suppliers, which relies on a standard laboratory X-ray source producing a beam with large angular divergence to deliver high geometric magnification. This approach can work well for small sample sizes, but, in practice this imposes physical sample size limitations due to the physics of such a design. [1] By implementing a novel X-ray detection system, mirroring the designs in place at synchrotron micro- and nano-TXM beamlines, sub-micron resolution is now routinely achievable in the laboratory as well with the Xradia VersaXRM across a large range of sample sizes (Figure 2). The lowered geometrical restrictions are met with a tunable contrast enhancement mechanism, which combines optimized scintillation technology for both absorption and propagation phase contrast, for a uniquely flexible laboratory X-ray microscope platform for modern in-situ and 4D studies on a wide variety of sample types. [1]

Here, we present the current state of the art in laboratory X-ray microscopes and their impact on 3D and 4D research across a number of specific applications including energy materials, composites and biomaterials. 
References:

[1] J. Gelb, "Functionality to Failure: Materials Engineering in the $4^{\text {th }}$ Dimension," Adv. Mat. Proc. 170(10), pp. 14-18, October 5, 2012.

[2] B. M. Patterson, K. Henderson, Z. Smith, D. Zhang, and P. Giguere, "Applications of Micro-CT to In-Situ Foam Compression and Numerical Modeling," Microscopy \& Analysis 26(2), S4-S7, 2012.

[3] Y. Feng, et al., "Nanofabrication of high aspect ratio $24 \mathrm{~nm}$ X-ray zone plates for X-ray imaging applications," J. Vac. Sci. Technol., B25, p 2004-7, 2007.

[4] A. Tkachuk, F. Duewer, H. Cui, M. Feser, S. Wang, and W. Yun, "X-ray Computed Tomography in Zernike Phase Contrast Mode at $8 \mathrm{keV}$ with 50-nm Resolution using Cu Rotating Anode X-ray Source," Z. Kristallogr. 222, pp. 650-655, 2007.
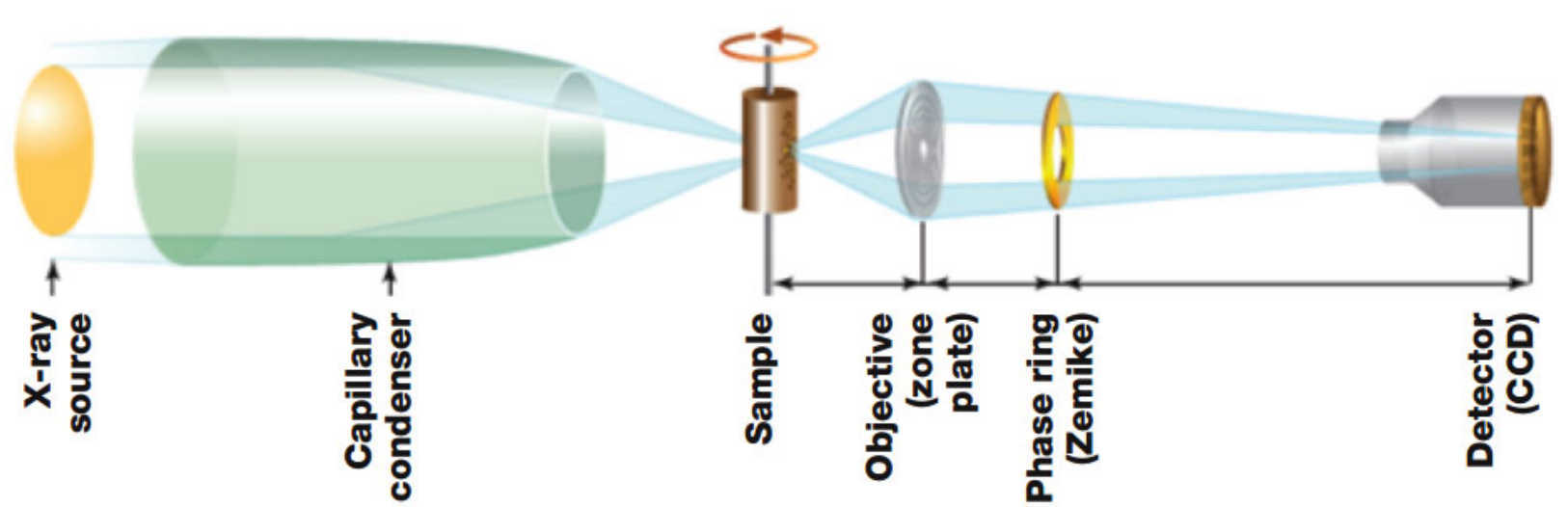

Figure 1. Schematic diagram of a true nano-scale laboratory XRM. This design is based on synchrotron developments, using X-ray focusing optics to achieve high spatial resolution.

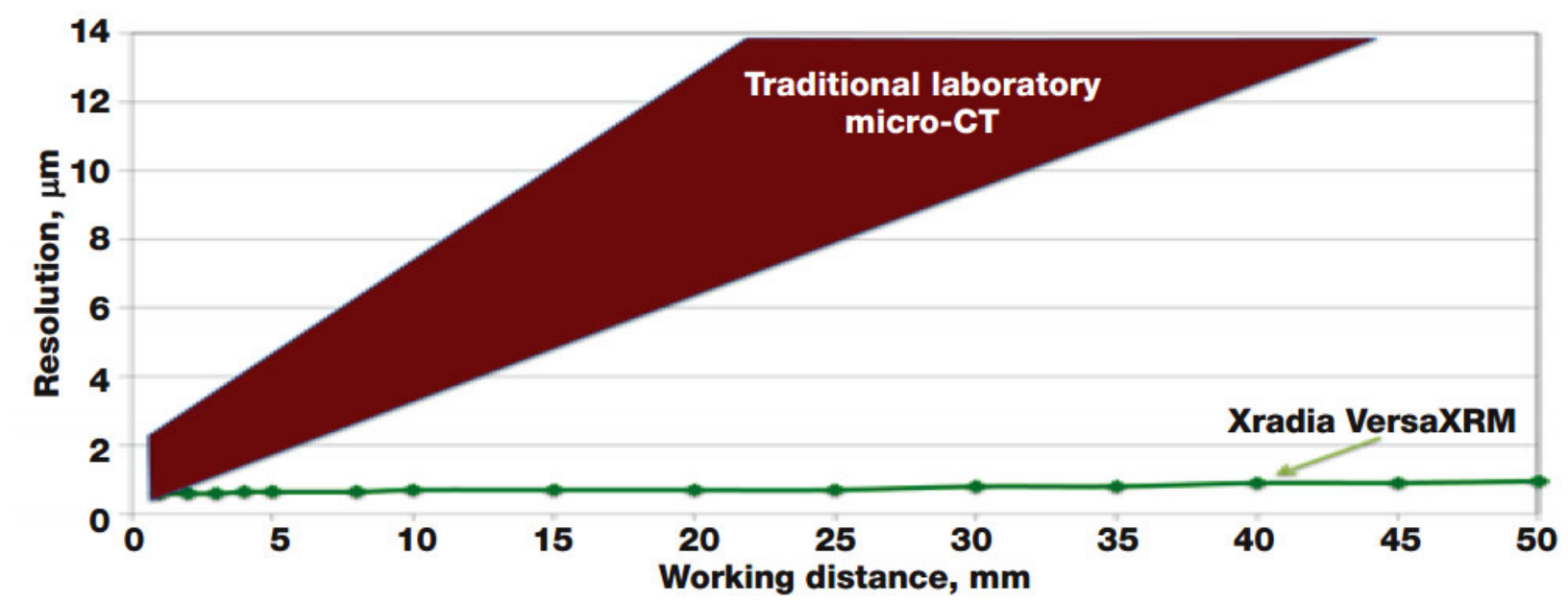

Figure 2. The synchrotron-style design of the laboratory VersaXRM has been shown to achieve high spatial resolution even with large sample sizes (working distance). 